\title{
Algoritmo para la detección automática de sangrados quirúrgicos utilizando visión por computador
}

\author{
A. García, J.M. Vicente, C. G. Juan, J.M. Sabater-Navarro \\ Universidad Miguel Hernández de Elche, alvaro.garciam@umh.es
}

\begin{abstract}
Resumen
El trabajo presenta el diseño y resultados obtenidos con un algoritmo de visión artificial para la detección automática de sangrados utilizando imágenes laparoscópicas o imágenes de mini-robots camarógrafos. El algoritmo se fundamenta en el cálculo de umbrales dinámicos en el espacio de color. Se muestran los resultados obtenidos con imágenes de videos de operaciones reales y con el sistema experimental desarrollado para este trabajo.
\end{abstract}

Palabras Clave: visión, sangrado, cirugía.

\section{INTRODUCCIÓN}

En cirugía mínimamente invasiva, las hemorragias siguen siendo una de las principales complicaciones presentes en todo tipo de intervenciones, siendo especialmente peligrosas las lesiones vasculares mayores (en inglés Major Vascular Injury, MVI) [1]. Aunque no existe una literatura extensa respecto a la incidencia de esta problemática, encontramos el estudio de Opitz et al [2] que realizaron analizando más de 43.000 intervenciones en clínicas y hospitales suizos, concluyendo que la incidencia de los sangrados en cirugías laparoscópicas ascendía al 1.7\% mientras que la incidencia de los MVI asciende al $0.09 \%$.

Respecto a la cirugía abdominal, más concretamente la colecistectomía laparoscópica, encontramos un estudio de Opasanon et al [3] que recopila varios estudios previos aportando unas incidencias en torno a $0.07-1.2 \%$. Especialmente interesante es el estudio de Duca et al [4], en el cual analiza las complicaciones surgidas en 9.542 colecistectomías laparoscópicas durante un periodo de 9 años, encontrando una incidencia de las complicaciones por hemorragia del $2.3 \%$, una conversión a cirugía abierta del $1.9 \%$ de las cuales un $4.8 \%$ fueron consecuencia directa de una hemorragia.

Aunque estos porcentajes puedan parecer bajos, se estima que entre el $10 \%$ y el $15 \%$ de la población de un país desarrollado será diagnosticado de piedras en la vesícula y requerirá muy probablemente una intervención quirúrgica. Esto significa que en un país como EEUU donde se estima que se realizan aproximadamente unas 750.000 colecistectomías al año, de las cuales el $90 \%$ se realizan mediante técnicas laparoscópicas, aproximadamente 15.500 personas sufrirán una hemorragia durante la intervención y 615 requerirán una conversión a cirugía abierta debido a esta complicación [5].

Las consecuencias de un sangrado en laparoscopía varían desde un simple retardo en la intervención, un mayor periodo de hospitalización, o incluso ser causa de mortalidad del paciente [6].

En general, todos los autores señalan la importancia de una rápida detección de una hemorragia para paliar sus efectos negativos. Por ello, se requiere de un sistema fiable capaz de analizar los factores determinantes de esta complicación y detectarla lo antes posible. En este trabajo se describe el diseño e implementación de un algoritmo de visión artificial que analice las imágenes recibidas directamente de una cámara laparoscópica y detecte tanto la presencia de charcos de sangre como el inicio de un sangrado masivo.

Aún a pesar de que no se ha encontrado en la bibliografía ningún intento de desarrollar un algoritmo de visión artificial para detectar un sangrado o sangre en una intervención de laparoscopía, sí que existe una extensa bibliografía centrada en el desarrollo de algoritmos para la clasificación de las imágenes obtenidas mediante una cápsula endoscópica con el objetivo de buscar hemorragias en el tracto gastrointestinal [7], [8].

Algunos autores se basan en la segmentación y clasificación de los píxeles según sus parámetros de los espacios de color HVS [9], [10], pero destaca especialmente el trabajo de Tonmoy Ghosh et al que clasifica las imágenes mediante un sencillo umbral que consiste en buscar imágenes con unos ratios globales $\mathrm{B} / \mathrm{R}$ y $\mathrm{G} / \mathrm{R}$ inferiores a 0.6 en el espacio de color BGR [11] Este fue el punto inicial del diseño de nuestro algoritmo.

\section{MATERIALES Y MÉTODOS}

\subsection{MATERIALES}


Para el desarrollo y testeo del algoritmo se han reunido dos series diferentes de videos. La primera serie está compuesta por videos de intervenciones realizadas mediante técnicas mínimamente invasivas tradicionales, grabadas usando una cámara laparoscópica directamente manipulada por un miembro del personal quirúrgico que incorpora una fuente de luz.

La otra serie está compuesta por videos grabados en un pelvitrainer de nuestro laboratorio mediante una cámara cenital y fuentes de luz independientes portados por mini robots magnéticamente fijados al "abdomen" del paciente desde el exterior y controlados mediante brazos robóticos. Para los experimentos se creó una sangre artificial movida mediante una bomba de agua (Figura 1).

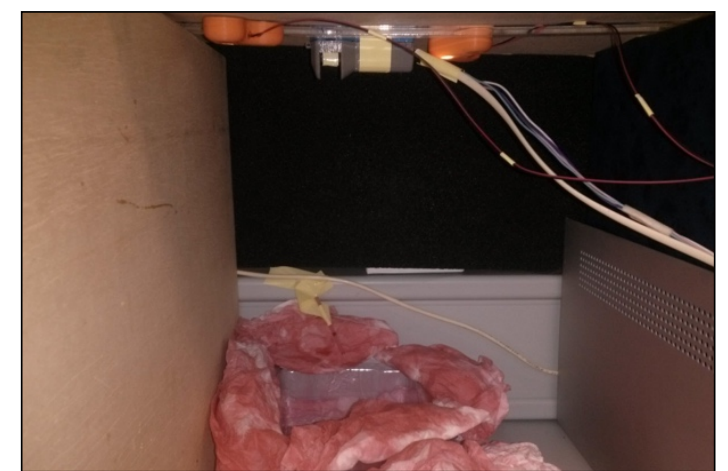

Figura 1: Set-up de experimentación usado para las pruebas con cámara cenital

Se grabaron 15 pruebas de cada uno de los 5 experimentos en los que se situaba la cámara en distintas posiciones y con distintos ángulos de visión, además de variar de un experimento a otro tanto la iluminación del entorno como las posiciones de las fuentes de luz y la cantidad de "tejido" manchado de rojo que simulan los órganos internos del paciente.

De cada una de las series de videos se tomaron una veintena de imágenes en distintos instantes de un sangrado y se crearon mapas binarios clasificando sus píxeles en sangre o fondo, usados para el cálculo de la efectividad del algoritmo.

\subsubsection{Algoritmo}

El primer paso del desarrollo del algoritmo consistió en comprobar la efectividad del algoritmo de Tonmoy Ghosh et al aplicándolo a varios videos de ambas series. Se pudo comprobar que de forma general los ratios globales $B / R$ y $G / R$ de los frames sufrían un descenso pronunciado al producirse un sangrado, pero que dependía fuertemente de la iluminación, la buena visibilidad y sobre todo del movimiento de la cámara. Con el objetivo de buscar el umbral óptimo para la segmentación de los píxeles de sangre, se analizaron todas las imágenes extraídas de los videos de ambas series probando umbrales desde 0.1 hasta 0.9 para segmentar los píxeles de sangre, cuyos resultados se pueden observar en las siguientes gráficas (Figura 2).
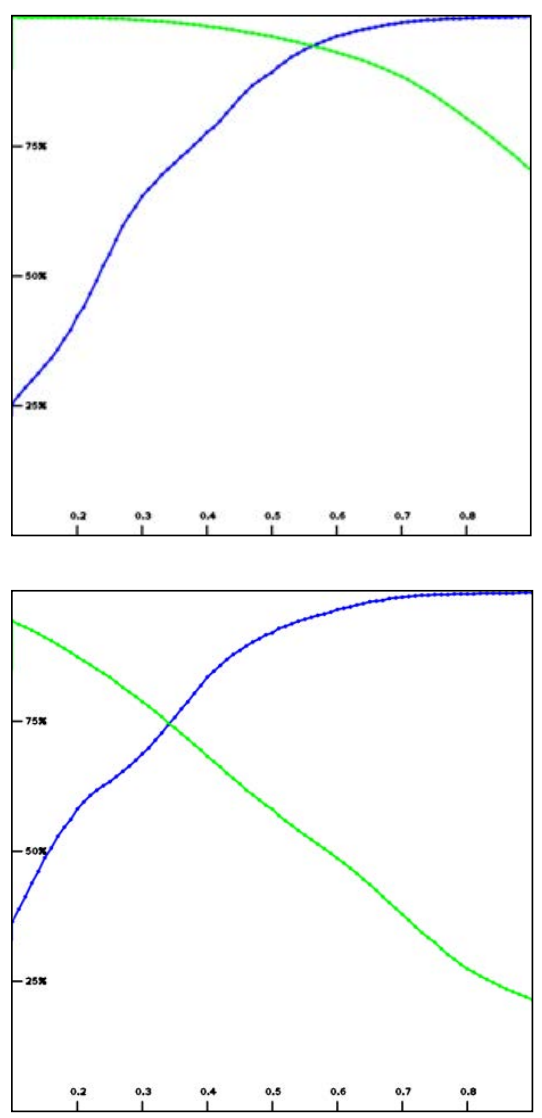

Figura 2: Sensibilidad (azul) y especificidad (verde) del análisis de las imágenes extraídas de los videos grabados mediante un mini robot cámara cenital (arriba) y una cámara laparoscópica tradicional (abajo).

Como se puede observar en las gráficas, se obtuvieron buenos resultados en las pruebas in vitro con un umbral de 0.54, manteniendo los valores de la sensibilidad y la especificidad por encima del $93 \%$. Sin embargo, los resultados con las imágenes in vivo no ofrecieron unos resultados satisfactorios, ya que el algoritmo tendía a confundir órganos internos de un tono rosado (por ejemplo el hígado) con sangre, produciendo un descenso muy pronunciado de la especificidad conforme aumentaba el valor del umbral.

A raíz de estas pruebas se concluyó que se requería de un sistema de umbrales dinámicos más restrictivo que fuera capaz de extraer de la imagen los píxeles de sangre sin confundirlos con píxeles de fondo y así evitar que el algoritmo ignore una posible hemorragia. Para ello se buscó una relación entre los niveles globales $\mathrm{B} / \mathrm{R}$ y $\mathrm{G} / \mathrm{R}$ de las imágenes in vitro y de cada uno de sus píxeles (Figura 3). 

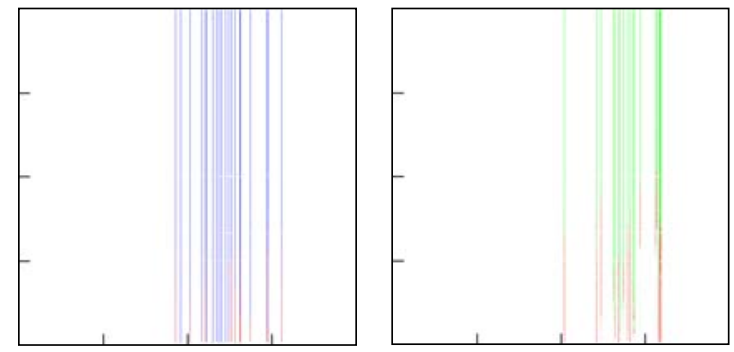

Figura 3: Proporciones B/R y G/R globales de la imagen en el eje de abscisas y los ratios $B / R$ y $G / R$

de cada pixel en el eje de ordenadas, donde los puntos rojos de las gráficas representan los píxeles de sangre y los azules o verdes representan los píxeles de fondo.

Como se observa, existe un decremento de las proporciones $\mathrm{B} / \mathrm{R}$ y $\mathrm{G} / \mathrm{R}$ de los píxeles conforme se reducen las proporciones globales de la imagen. Se decide probar una segmentación de los píxeles según las siguientes ecuaciones gráficamente representadas (Figura 4).

$$
\begin{aligned}
& \operatorname{Umbral}_{\mathrm{B} / \mathrm{R}}=0^{\prime} 625 \cdot \operatorname{ratio}_{\mathrm{B} / \mathrm{R}}+0^{\prime} 0125 \\
& \operatorname{Umbral}_{\mathrm{G} / \mathrm{R}}=0^{\prime} 625 \cdot \text { ratio }_{\mathrm{G} / \mathrm{R}}+0^{\prime} 0125
\end{aligned}
$$
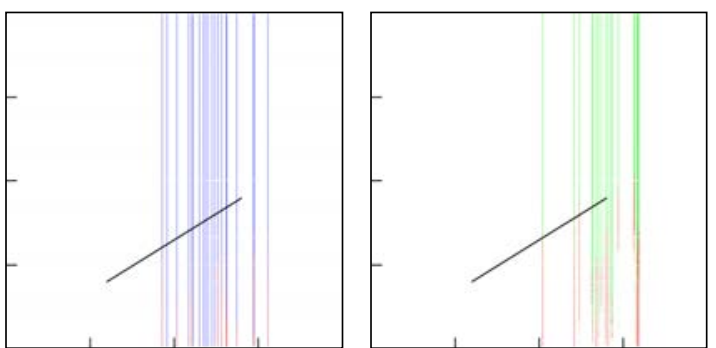

Figura 4: Gráficas de la figura 3 con una recta superpuesta que representa el umbral dinámico usado en este algoritmo.

El objetivo de esta segmentación es reducir al máximo el número de falsos positivos producidos por el algoritmo al detectar píxeles de sangre. Mediante estos umbrales se analizaron las imágenes usadas para crear las gráficas, segmentando los píxeles según si sus ratios individuales son ambos menores al umbral establecido (píxeles de sangre) o mayores (píxeles de fondo). Tras la segmentación se realizaron unas transformaciones morfológicas de erosión y dilatación para eliminar píxeles sueltos que sin lugar a dudas no forman parte de un charco de sangre. La figura 5 esquematiza el algoritmo utilizado.

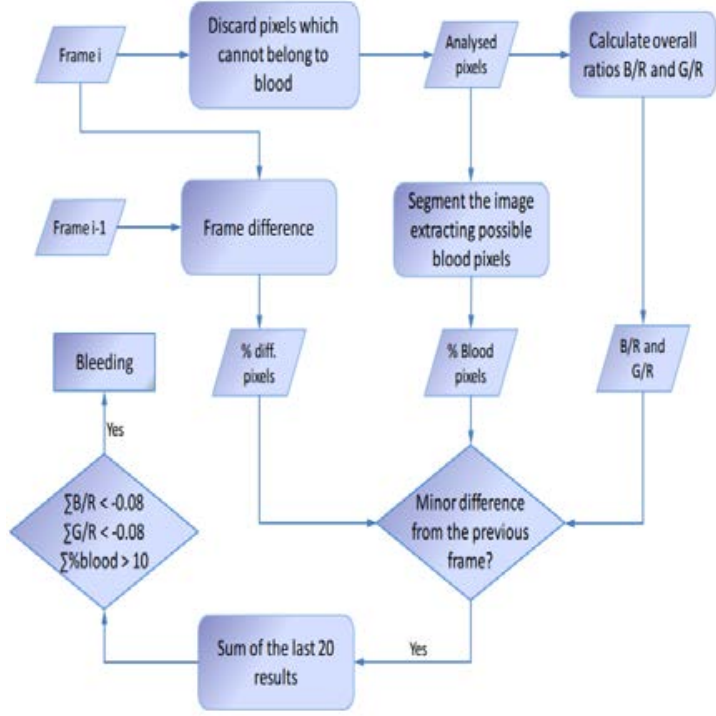

Figura 5: Esquema del algoritmo.

\section{EXPERIMENTACION}

Finalmente se compararon los resultados del análisis con los mapas binarios que marcan los píxeles que realmente pertenecen a sangre, obteniendo para este algoritmo una sensibilidad de $80{ }^{\prime} 26 \%$ y una especificidad de $98^{\prime} 41 \%$.

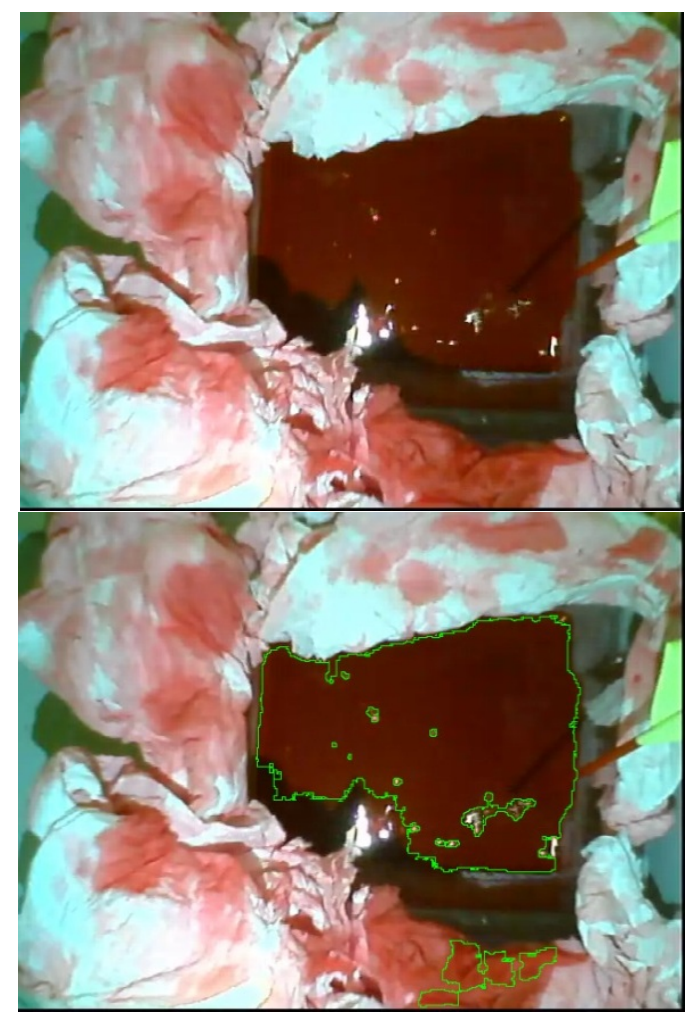

Figura 6: Imagen original extraída de una prueba in vitro (arriba) y la imagen resultante del análisis con los píxeles de sangre detectados rodeados mediante una línea verde (abajo). 
También se analizó la influencia de la luz sobre el algoritmo, descubriéndose tan perjudicial una luz cenital cercana a la cámara que cree reflejos como una iluminación pobre que no otorgue a los píxeles de sangre la tonalidad adecuada.

El siguiente paso consistió en el análisis de imágenes in vivo, donde debido a la alta variación de la iluminación, la presencia del gas generado por la cauterización y al movimiento brusco de las cámaras, los resultados obtenidos fueron inferiores con una sensibilidad de $69^{\prime} 46 \%$ y una especificidad de 94’21\%.
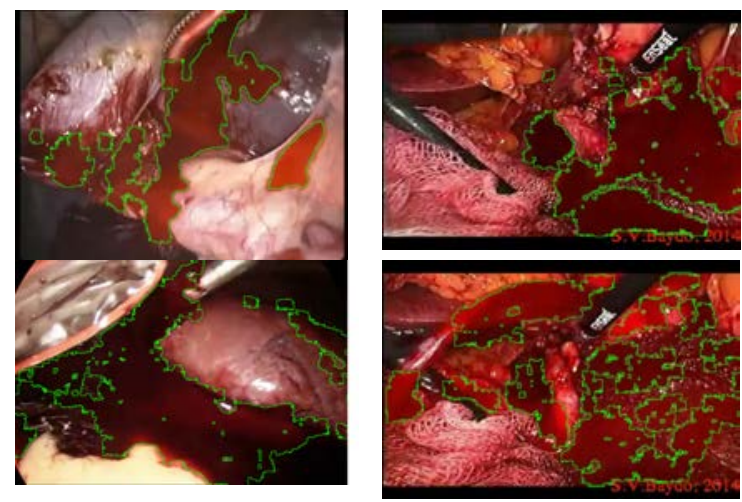

Figura 7. Resultados del análisis de imágenes de operaciones reales, donde los píxeles de sangre detectados se muestran rodeados mediante una línea verde.

Si se comparan estos resultados con los obtenidos al inicio del este documento mediante umbrales estáticos, se observa claramente una mejoría importante para las imágenes extraídas de videos de operaciones reales, manteniendo una especificidad relativamente alta (Figura 8 ).

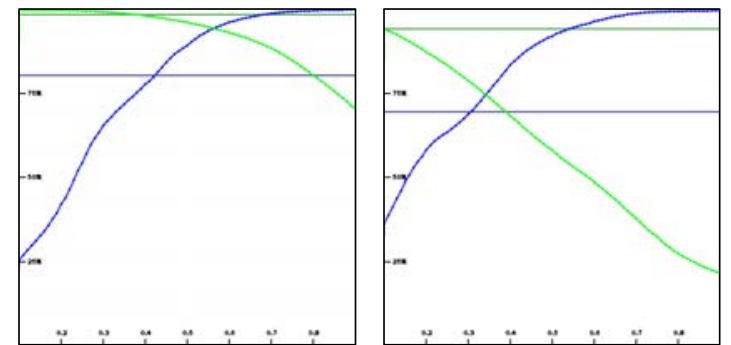

Figura 8: Sensibilidad (azul) y especificidad (verde) del análisis de las imágenes extraídas de los videos grabados mediante un mini robot cámara cenital (izquierda) y una cámara laparoscópica tradicional (derecha). Las curvas representan los resultados del análisis mediante el algoritmo de Tonmoy Ghosh et al mientras que las rectas representan los resultados del análisis mediante el umbral dinámico descrito en este documento.
Los casos donde mejor se aprecian las ventajas de los umbrales dinámicos frente los estáticos es en imágenes donde el entorno quirúrgico esté completamente manchado de sangre, de donde se pretenden segmentar solo los píxeles que pertenecen a la sangre que está siendo expulsada en ese momento por la incisión del sangrado.
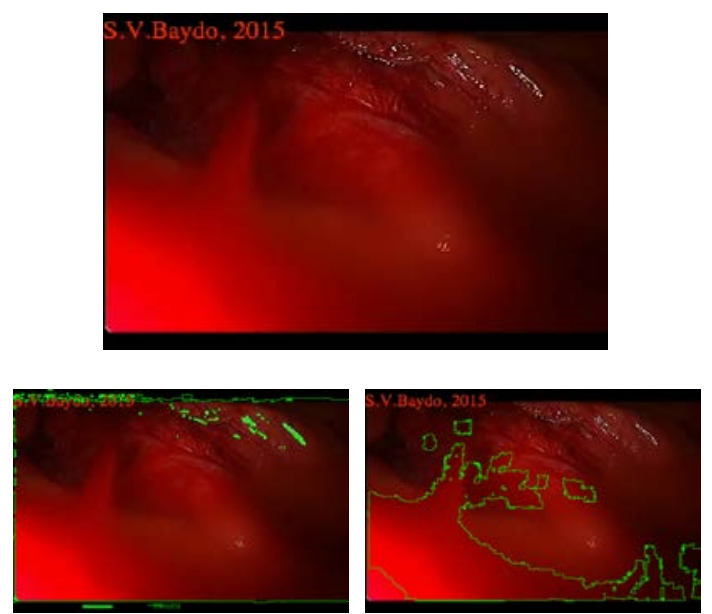

Figura 8. Comparativa del análisis de una imagen de una operación real (arriba) mediante un umbral estático de 0.6 (izda.) y un umbral dinámico (der).

A continuación se pasó a analizar los videos completos de los experimentos in vitro, graficando los valores respecto al tiempo de tanto el porcentaje de píxeles de sangre respecto al total como las proporciones medias globales de $B / R$ y $G / R$. Se observó que para un sangrado típico la mayor variación de estos valores (aumento del porcentaje de píxeles de sangre y disminución de los ratios $\mathrm{B} / \mathrm{R}$ y $\mathrm{G} / \mathrm{R})$ se daba en un periodo de tiempo de aproximadamente un segundo, por lo que se midieron las variaciones totales durante un periodo de 20 frames alrededor del instante en el que se produce el sangrado. Para ello se realizaron 5 experimentos diferentes con 15 pruebas en cada uno, variando dentro de cada experimento la posición de la cámara cenital y de las fuentes de luz. Los resultados fueron los siguientes:

Tabla 1: datos experimentales.

\begin{tabular}{|l|l|l|l|}
\hline & $\begin{array}{l}\text { Pendiente B/R } \\
\text { mín }\end{array}$ & $\begin{array}{l}\text { Pendiente G/R } \\
\text { mín }\end{array}$ & $\begin{array}{l}\text { Pendiente } \\
\text { sangre máx }\end{array}$ \\
\hline $\begin{array}{l}\text { Test in } \\
\text { vitro A }\end{array}$ & -0.06633084 & -0.069726273 & 11.325468 \\
\hline $\begin{array}{l}\text { Test in } \\
\text { vitro B }\end{array}$ & -0.1571744 & -0.157906 & 15.5642533 \\
\hline $\begin{array}{l}\text { Test in } \\
\text { vitro C }\end{array}$ & -0.122537159 & -0.1088030912 & 13.0489059 \\
\hline $\begin{array}{l}\text { Test in } \\
\text { vitro D }\end{array}$ & -0.089034813 & -0.070206127 & 11.5687333 \\
\hline $\begin{array}{l}\text { Test in } \\
\text { vitro E }\end{array}$ & -0.108281273 & -0.077005013 & 10.6634087 \\
\hline $\begin{array}{l}\text { Media } \\
\text { total }\end{array}$ & -0.108671697 & -0.096734865 & 12.4341538 \\
\hline
\end{tabular}


Finalmente se analizaron una serie de 20 videos de operaciones reales (en los cuales algunos muestran un sangrado masivo mientras otros muestran una intervención sin incidentes) estableciendo como condiciones para detectar un sangrado:

- Suma de las pendientes $\mathrm{B} / \mathrm{R}$ de los últimos 20 frames inferior a -0.08 .

- Suma de las pendientes $\mathrm{G} / \mathrm{R}$ de los últimos 20 frames inferior a -0.08 .

- Suma de las pendientes del porcentaje de sangre de los últimos 20 frames superior a 10.

Se obtuvieron unos resultados satisfactorios, obteniendo para el algoritmo final una sensibilidad de $76{ }^{\prime} 47 \%$ y una especificidad de $83{ }^{\prime} 33 \%$.

\section{Conclusiones}

Hoy en día las hemorragias siguen siendo una de las posibles complicaciones que pueden ocurrir durante una operación mediante técnicas mínimamente invasivas y las limitaciones visuales impuestas por el uso de una cámara laparoscópica dificultan su detección y tratamiento, agravando una situación ya de por si peligrosa.

En este documento se presentan los primeros pasos para el diseño de un algoritmo de visión artificial mediante el cual detectar un posible sangrado masivo solo mediante el análisis de las imágenes recibidas directamente de una cámara laparoscópica. Los resultados obtenidos son aceptables, si bien dependen directamente de la calidad de la imagen, del movimiento de la cámara, del uso de un cauterizador o bisturí eléctrico cerca de la cámara o de los cambios de iluminación.

Como siguiente paso, el algoritmo requiere de una optimización para aumentar su efectividad, por ejemplo robusteciéndolo frente a variaciones en la iluminación. También se podrá fusionar este algoritmo detector de hemorragias con otro diseñado para la detección y tracking de gasas quirúrgicas, ya que ambos elementos están fuertemente asociados.

\section{Agradecimientos}

Los autores quieren agradecer el apoyo económico del Consejo Nacional de Investigación de España a través del proyecto DPI2013-47196-C3-2-R. Carlos G. Juan agradece al Ministerio de Educación, Cultura $\mathrm{y}$ Deporte (MECD) del Gobierno de España su apoyo a través del Programa de Formación del Profesorado Universitario (FPU).
[1]. Robin Kaushik, "Bleeding complications in laparoscopic cholecystectomy: Incidence, mechanisms, prevention and management", 2010.

[2]. Opitz et al., "Bleeding remains a major complication during laparoscopic surgery: analysis of the SALTS database", 2005.

[3]. Opasanon et al., "Major Vascular Injury in Laparoscopic Cholecystectomy", 2011.

[4]. Duca et al., "Laparoscopic cholecystectomy: incidents and complications. A retrospective analysis of 9542 consecutive laparoscopic operations", 2003.

[5]. Laura M. Stinton and Eldon A. Shaffer, "Epidemiology of Gallbladder Disease: Cholelithiasis and Cancer", 2012.

[6]. Anna Mases et al., "Injury to the Abdominal Aorta During Laparoscopic Surgery: An Unusual Presentation", 2000.

[7]. Jackson D. Hamilton et al, "Multidetector CT Evaluation of Active Extravasation in Blunt Abdominal and Pelvic Trauma Patients", 2008.

[8]. Jürgen K. Willmann, "Multidetector CT Detection of Active Haemorrhage in Patients with Blunt Abdominal Trauma", 2002.

[9]. Phooi Yee Lau and Paulo Lobato Correia, "Detection of bleeding patterns in WCE video using multiple features", 2007.

[10]. Giritharan et al., "Bleeding Detection from Capsule Endoscopy Videos", 2008.

[11]. Tonmoy Ghosh et al., "Automatic Bleeding Detection in Wireless Capsule Endoscopy Based on RGB Pixel Intensity Ratio", 2014.

\section{Referencias}

